\title{
MODELLING THE RATE- AND TEMPERATURE-DEPENDENT MICRO-MECHANICAL BEHAVIOUR OF CARBON FIBER REINFORCED PVDF
}

\author{
$\underline{\text { T. Lenders }}^{1 *}$, J.J.C. Remmers ${ }^{1}$, T. Pini ${ }^{1}$, L.E. Govaert ${ }^{1}$ and M.G.D. Geers $^{1}$ \\ ${ }^{1}$ Department of Mechanical Engineering, Eindhoven University of Technology \\ *t.lenders@tue.nl
}

The conditions to which fiber reinforced plastics (FRPs) are exposed in state of the art applications are becoming more extreme, for example in the offshore oil and gas industry. Therefore, the ability to predict the long-term behaviour, and thereby identifying the failure mechanisms, of fiber reinforced plastics is of great importance. Especially under these extreme conditions, the contribution of the matrix plays an important role and a detailed description of its behaviour is required. In oil and gas applications, polyvinylidene fluoride (PVDF) is used because of its excellent gas barrier properties. In this work the rate- and temperature-dependent micromechanical behaviour of carbon fiber reinforced polyvinylidene fluoride is studied.

The behaviour of the composite is studied by using a micro-mechanical model that is composed of individually modelled carbon fibers embedded in a PVDF matrix. The time- and temperaturedependent behaviour of PVDF is captured by the Eindhoven Glassy Polymer (EGP) constitutive model [1]. This model enables the description of the intrinsic behaviour of the semi-crystalline matrix over a range of applied strain rates and temperatures using a single set of material parameters. The characterization of these material parameters, requires a set of experimental data obtained from uniaxial compression and tensile tests performed at different temperatures and applied strain rates. To describe the material behaviour of the individually modelled carbon fibers, an elastic orthotropic material model is employed. Off-axis tensile tests of the composite led to the observation that the interface behaviour between matrix and fiber must be incorporated in the micro-mechanical model as well. Subsequently, an interface between the matrix and fiber is added to the model by using cohesive zone interface elements. The behaviour of these interface elements is described by an appropriate constitutive relation.

It is demonstrated what influence the rate- and temperature dependence of PVDF, described by the EGP-model, and the behaviour of the interface elements have on the micro-mechanical response of the composite.

\section{References}

[1] L.C.A. van Breemen, T.A.P. Engels, E.T.J. Klompen, D.J.A. Senden and L.E. Govaert (2012). Rate- and temperature-dependent strain softening in solid polymers. Journal of Polymer Science, Part B: Polymer Physics, 50(24), 1757-1771. 